\title{
Pt Nanoparticles Supported on Nitrogen doped Carbon as an Efficient Catalyst for Decalin Dehydrogenation
}

\author{
Huiru Y un, Zhuo Li, Shiguang Fan, Jian Wang* and He Liu* \\ State K ey Laboratory of Heavy Oil Processing, College of Chemical Engineering, China University of Petroleum (East China), Qingdao \\ 266580, China.
}

\begin{abstract}
A novel Pt/CN catalyst was synthesized by sodium borohydride treatment. The physical and chemical properties of Pt/CN catalyst were characterized by $X$-ray diffraction (XRD), brunner-emmet-teller (BET), transmission electron microscope (TEM) and High-resolution transmission electron microscopy (HRTEM). The characterized results showed that the catalyst has a high specific surface area, mesoporous structure and the mean size of Pt nanoparticles is $2.59 \mathrm{~nm}$. Subsequently, the catalytic performance of $\mathrm{Pt} / \mathrm{CN}$ catalyst for decline dehydrogenation was studied. Pt/CN catalyst exhibited excellent performance in decalin dehydrogenation with the conversion of decalin was $30.70 \%$, and the selectivity of naphthalene was $90.86 \%$ at $200{ }^{\circ} \mathrm{C}$ for 150 minutes. When the reaction temperature increased to $210{ }^{\circ} \mathrm{C}$, the conversion of catalyst increased to $52.02 \%$, and the selectivity of naphthalene reduced to $90.21 \%$. The possible reason may be attributed to the difficulty in converting decalin to tetralin. This paper would provide a novel method for the synthesis of efficient dehydrogenation catalyst of decalin..
\end{abstract}

\section{Introduction}

Hydrogen energy has attracted wide attention because of its environmental friendliness, recyclability and high combustion calorific value. It is considered to be one of the best alternatives to traditional fuels. However, hydrogen is easy to leak and explode at ambient temperature, which making it difficult to store and transport. In order to solve this problem, many hydrogen storage technologies have been studied, such as highpressure gaseous hydrogen storage, liquefied hydrogen storage, metal alloy hydrogen storage and organic liquid hydrogen storage [1-3]. Decalin has been widely favored as an organic liquid hydrogen storage material due to its high hydrogen storage capacity $(7.3 \mathrm{wt} \%)$ and inexpensive price. As a strong endothermic reaction, the reaction of decalin dehydrogenation requires to be carried out at high temperature, which makes the catalyst easy to coking and deactivation $[4,5]$. Therefore, it is urgent to develop an efficient catalyst for decalin dehydrogenation.

Noble metals (such as Pt, Pd, Rh, Ir) were used as active component for decalin dehydrogenation [6,7]. Among them, Pt was widely used because of its high catalytic activity and oxidation resistance. To further improve the catalytic activity of catalyst for decalin dehydrogenation, a series of attempts have been made. One method to improve the dehydrogenation activity of decalin was added the second component (such as W, Re, Ir) to prepare bimetallic catalyst [6,7]. Another method to improve the dehydrogenation performance of decalin was regulated the catalyst supports, such as $\mathrm{Al}_{2} \mathrm{O}_{3}$ and carbon-based support [8-11]. Carbon-based support was one of the commonly used catalyst support for decalin dehydrogenation, which surface could be controlled and modified by the functional groups. The dehydrogenation performance of decalin was affected by the type of carbon materials, pore structure and specific surface area. $\mathrm{Li}$ and coworkers [9] studied the effect of carbon nanofiber (tubular (t-CNFs), fishbone (f-CNFs) and plate (p-CNFs)) supports with different morphologies on the dehydrogenation performance of decalin, and found that the best dehydrogenation performance of decalin was obtained by using the p-CNFs as the support. Sebastián and coworkers [10] pointed out that the catalyst with large specific surface area can obtain better initial activity, the small pore size would hinder the mass transfer of the products, thus reducing the dehydrogenation activity of decalin. Lee and coworkers [11] found that $\mathrm{Pt}$ nanoparticles could be dispersed effectively by oxidation treatment of carbon support, which can improve the dehydrogenation activity of decalin. Therefore, a fine carbon support was important to improve the dehydrogenation performance of decalin.

In this paper, a novel nitrogen-doped carbon supported Pt catalyst with large specific surface area and pore size was synthesized. The experimental results showed that the $\mathrm{Pt} / \mathrm{CN}$ catalyst exhibited excellent activity of decalin dehydrogenation. The conversion of decalin was $30.70 \%$ and $50.02 \%$ at $200-210^{\circ} \mathrm{C}$ for 150 minutes, respectivly.The catalyst physical and chemical property was obtained by XRD, BET, TEM and HRTEM.

* Corresponding author: Wang: 386426087@ qq.com Liu: aoyun3002008@126.com 


\section{Experimental}

\subsection{Materials}

Decalin $\left(\mathrm{CH}_{10} \mathrm{H}_{18}\right)$, chitosan $\left(\left(\mathrm{C}_{6} \mathrm{H}_{11} \mathrm{NO}_{4}\right) \mathrm{N}\right)$, melamine $\left(\mathrm{C}_{3} \mathrm{H}_{3}\left(\mathrm{NH}_{2}\right)_{5}\right)$, chloroplatinic acid hexahydrate $\left(\mathrm{H}_{2} \mathrm{PtCl}_{6} \cdot 6 \mathrm{H}_{2} \mathrm{O}\right)$, sodium borohydride $\left(\mathrm{NaBH}_{4}\right)$, sodium hydroxide $(\mathrm{NaOH})$ and methanol $\left(\mathrm{CH}_{3} \mathrm{OH}\right)$ were purchased from Shanghai Chemical Reagent Company. $\mathrm{SiO}_{2}$ colloidal was purchased from Aldrich. All reagents were used as received and without any other purification.

\subsection{Catalysts Preparation}

The first step was to synthesize nitrogen-doped carbon support. Under specific experimental conditions, Chitosan (1 g), melamine $(1 \mathrm{~g})$ and silica colloid $(4 \mathrm{~mL})$ were mixed evenly in $20 \mathrm{ml} \mathrm{H}_{2} \mathrm{O}$, then added into a $50 \mathrm{ml}$ round-bottom flask, and evaporated at $100^{\circ} \mathrm{C}$. After drying, the product was annealed at $900^{\circ} \mathrm{C}$ in nitrogen atmosphere for 3 hours. The nitrogen-doped carbon support was obtained by Etching of sodium hydroxide and washing to neutrality. The second step is to prepare $\mathrm{Pt} / \mathrm{CN}$ catalyst. $\mathrm{H}_{2} \mathrm{O}(20 \mathrm{~mL}), \mathrm{CN}$ supports $(200 \mathrm{mg})$, and $\mathrm{H}_{2} \mathrm{PtCl}_{6} .6 \mathrm{H}_{2} \mathrm{O}(320 \mu \mathrm{L}, 0.1 \mathrm{~g} / \mathrm{mL})$ were added into a $50 \mathrm{ml}$ round bottom flask, respectively, then stired it at room temperature for 24 hours. The dispersion was reduced by Sodium borohydride. Finally, the product was centrifuged, washed and dried.

\subsection{Catalysts Characterizations}

The crystal structure of nitrogen-doped carbon supported platinum catalyst was analyzed by X-ray diffraction (XRD). The specific surface area of the catalyst was studied by brunner-emmet-teller (BET). The microstructure of the catalyst was studied by transmission electron microscope (TEM) and Highresolution transmission electron microscopy (HRTEM).

\subsection{Catalytic Dehydrogenation of Decalin}

Catalyst $(150 \mathrm{mg})$ and Decahydronaphthalene $(330 \mathrm{mg})$ were added into the reaction tube. The air of the reaction tube was changed to argon. Then, the reaction is carried out at in an oil bath. The gas and liquid products were analyzed by Gas chromatograph (GC).

\section{Results and Discussion}

\subsection{Physicochemical properties of $\mathrm{Pt} / \mathrm{CN}$ catalyst}

The XRD pattern of $\mathrm{CN}$ and $\mathrm{Pt} / \mathrm{CN}$ were shown in Fig. 1. The strong $\mathrm{C}(002)$ diffraction peak on $\mathrm{CN}$ pattern appeared at $24.39^{\circ}$. Compared with $\mathrm{CN}$ pattern, four distinct diffraction peaks appeared at $39.7^{\circ}, 46.24^{\circ}$, $67.45^{\circ}$ and $81.29^{\circ}$, which belong to the $\operatorname{Pt}(111), \operatorname{Pt}(200)$,
$\mathrm{Pt}(220)$ and $\mathrm{Pt}(311)$. It indicated that $\mathrm{Pt} / \mathrm{CN}$ catalyst has been successfully synthesized.

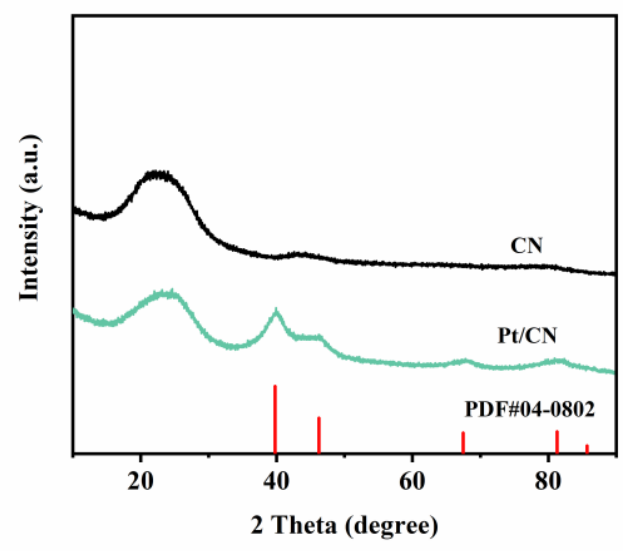

Fig. 1. XRD patterns of $\mathrm{CN}$ and $\mathrm{Pt} / \mathrm{CN}$ catalyst

The structural properties were characterized by BET, and the results were shown in Fig. 2. Pt/CN catalyst had large specific surface area and mesoporous structure. The specific surface area of $\mathrm{Pt} / \mathrm{CN}$ catalyst was 850.86 $\mathrm{m}^{2} \cdot \mathrm{g}^{-1}$ and the pore size was $10.1 \mathrm{~nm}$.
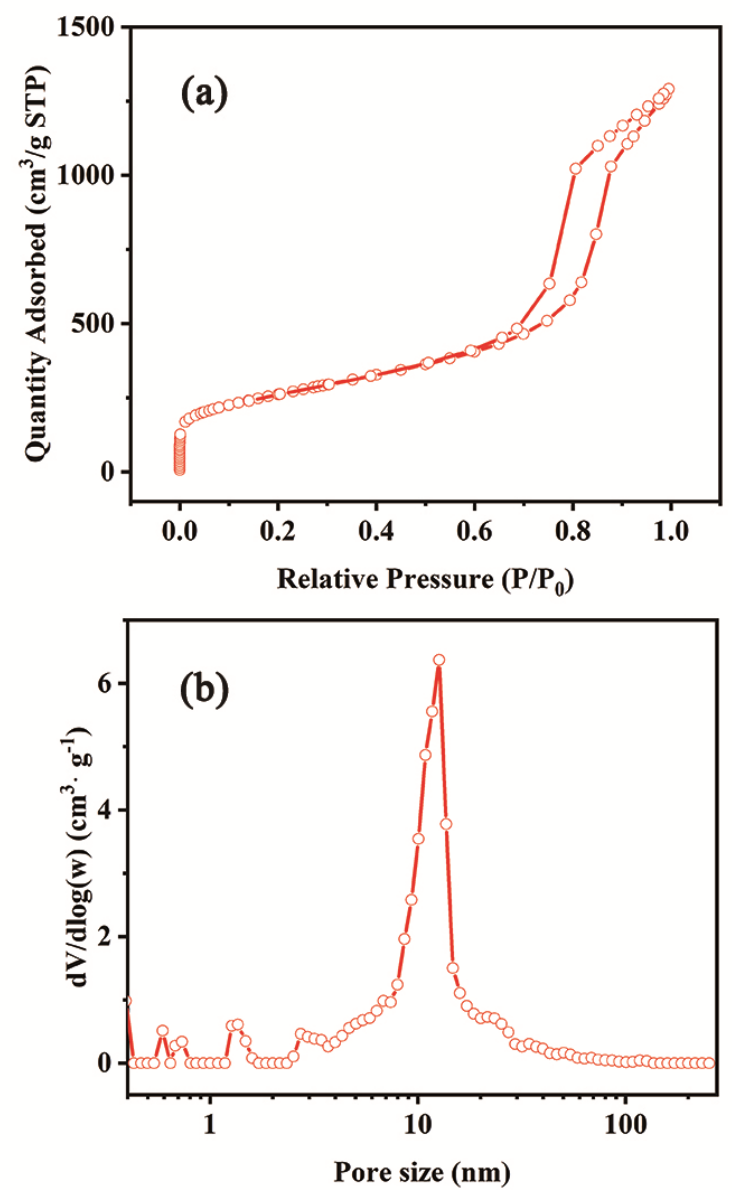

Fig. 2. The $\mathrm{N}_{2}$ adsorption/desorption isotherms and pore size distribution curves of $\mathrm{Pt} / \mathrm{CN}$ catalyst

The morphology and Pt size distribution of the catalyst was shown in Fig. 3. Fig. 3(a) and (b) showed the surface of catalyst had a lot of pore structures and $\mathrm{Pt}$ 
particles were evenly distributed on the support. To investigate the size of Pt particles, $200 \mathrm{Pt}$ particles in Fig 3(b) were counted. Fig. 3(d) showed the size of Pt particles were in the range of $1.75 \sim 3.6 \mathrm{~nm}$, and the average size of Pt nanoparticles was $2.59 \mathrm{~nm}$. As shonwn in Fig. 3(c), the lattice stripe gap of Pt particles were uniform and the spacing of lattice stripe was 0.226 $\mathrm{nm}$ corresponding to the crystal plane of Pt (111).
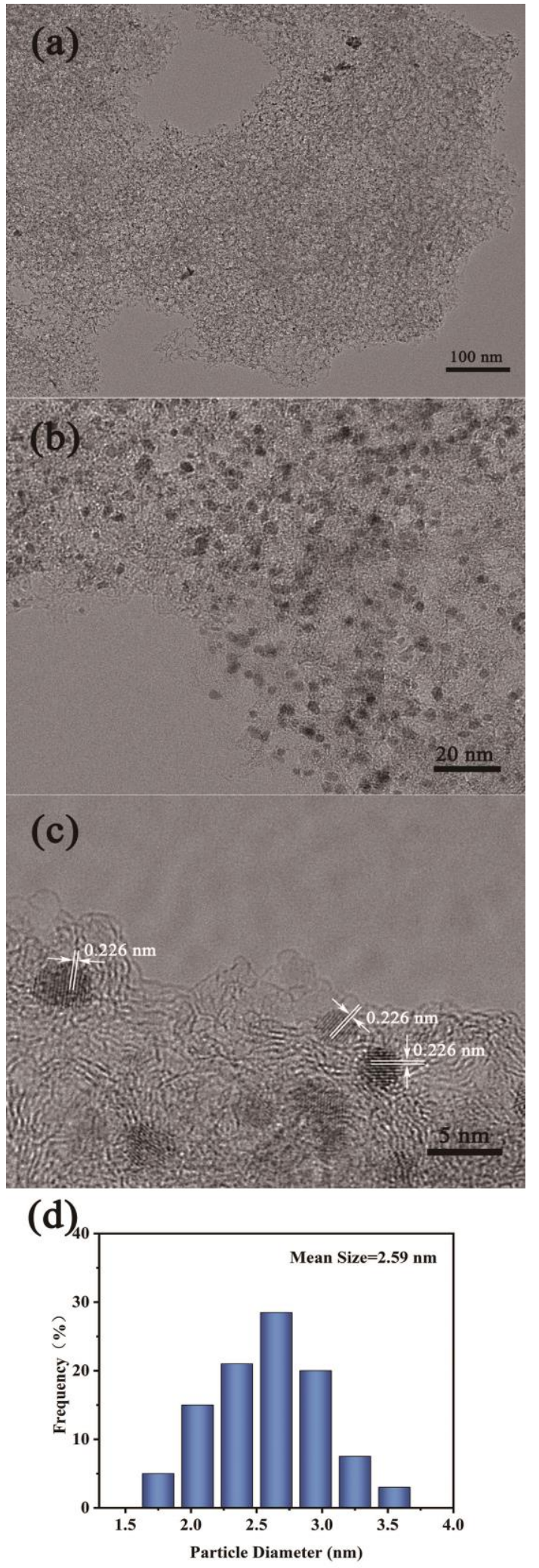

Fig. 3. (a) and (b) TEM images of Pt/CN catalyst; (c) HR TEM image of $\mathrm{Pt} / \mathrm{CN}$ catalyst; (d) the size distribution of Pt particles

\subsection{Catalytical activity}
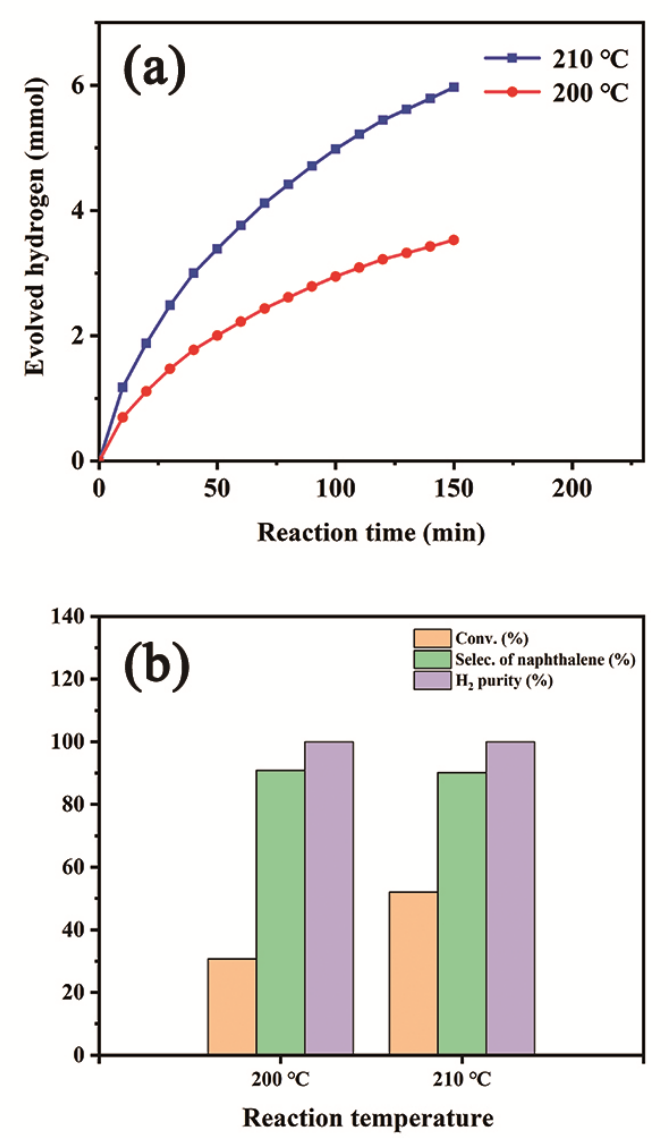

Fig. 4. Catalytic performances of $\mathrm{Pt} / \mathrm{CN}$ catalyst

The catalytic performances of $\mathrm{Pt} / \mathrm{CN}$ catalyst over dehydrogenation of decalin were shown in Fig. 4..The experimental results showed that the $\mathrm{Pt} / \mathrm{CN}$ catalyst exhibits excellent decalin dehydrogenation activity with the conversion rate of $30.70 \%$ and the selectivity rate of $90.86 \%$ at $200{ }^{\circ} \mathrm{C}$. At $210^{\circ} \mathrm{C}$ the conversion rate was $52.02 \%$ and the selectivity was $90.21 \%$. With the decrease of temperature, the selectivity rate of naphthalene increases slightly. It indicated that it was easier for tetralin to be dehydrogenated than decalin.

\section{Conclusions}

In this work, a mesoporous $\mathrm{Pt} / \mathrm{CN}$ catalyst with large specific surface area was successfully synthesized by sodium borohydride reduction. The specific surface area of the catalyst was $850.86 \mathrm{~m}^{2} ¥ \mathrm{~g}^{-1}$ and pore size of the catalyst was $10.1 \mathrm{~nm}$. Pt nanoparticles were dispersed well on the surface of support with the mean size of 2.59 $\mathrm{nm}$. The $\mathrm{Pt} / \mathrm{CN}$ catalyst exhibited excellent catalytic performance for decalin dehydrogenation. $\mathrm{Pt} / \mathrm{CN}$ catalyst catalyzed decalin dehydrogenation with the conversion rate was $30.70 \%$ (selectivity of naphthalene was $90.86 \%$ ) 
at $200{ }^{\circ} \mathrm{C}$ and $52.02 \%$ (selectivity of naphthalene was $90.21 \%$ ) at $210{ }^{\circ} \mathrm{C}$. This paper would provide a new strategy for the synthesis of efficient catalyst for decalin dehydrogenation.

\section{Acknowledgement}

This work was supported by the National Natural Science Foundation of China (Grant 21908248), the Fundamental Research Funds for the Central Universities (Grant 19CX02013A), the Provincial Natural Science Foundation of Shandong (Grant ZR2017BB021), the Provincial Postdoctoral Research Founding of Shandong (201702028), the State Key Laboratory of Heavy Oil Processing (SLKZZ-2017003).

\section{References}

1. S. Orimo, Y. Nakamori, et al. Chem. Rev. 107, 4111 (2007).

2. U. Eberle, M. Felderhoff, F. Schuth. Angew. Chem. Int. Ed. 48, 6608 (2009).

3. R.H. Crabtree. Energy Environ. Sci. 1, 134 (2008)

4. A.F. N obuko Kariya, M asaru Ichikawa. A ppl. Catal. A: Gen. 233, 91 (2002).

5. N. Jiang, K.S.R. Rao, et al.. Appl. Catal. A: Gen. 425, 62 (2012).

6. S. Hodoshima, H. A rai, Y. Saito. Int. J. Hydrogen Energ. 28, 197 (2003).

7. S. Hodoshima, S. Takaiwa, et al. Appl. Catal. A: Gen. 283, 235 (2005).

8. N. Kariya, A. Fukuoka, et al. A ppl. Catal. A: Gen. 247, 247 (2003).

9. P. Li, Y. Huang, et al. Catal. Commun. 10, 815 (2009).

10. D. Sebastián, C. Alegre, et al. Int. J. Hydrogen Energ. 39, 4109 (2014).

11. G. Lee, Y. Jeong, et al. Catal. Commun. 67, 40 (2015). 